\title{
Recent Advances in Materials and Technologies for Body-Centric and IoT Antenna Systems
}

\author{
S. Agneessens, S. Lemey, and H. Rogier \\ Ghent University - imec, IDLab, Department of Information Technology
}

\begin{abstract}
In the recent decade, we have witnessed a revolution in wireless communication systems. In this contribution, some novel materials and technologies for the development of dedicated antennas systems for body-centric communication and the Internet of Things are presented. In particular, the use of textile and cork materials as antenna substrates, combined with conducting electro-textiles or very thin copper-on-polyimide layers. Next, we focus on antenna topologies that yield highly stable radiation characteristics owing to their excellent body-antenna isolation. Therefore, we implement wide band cavity-backed slot antennas in substrate integrated waveguide technology. This enables the design of highly efficient active textile antenna systems where electronic circuitry and energy harvesters are deployed in very close proximity of the radiating parts of the antenna. Finally, we demonstrate how these systems may leverage multi-antenna processing techniques to increase throughput and robustness of the wireless channel.
\end{abstract}

Index Terms - Body-centric communication, Internet of Things (IoT), Substrate Integrated Waveguide (SIW), Active Antenna Systems, Cavity-Backed Slot Antenna.

\section{INTRODUCTION}

The recent introduction of novel wireless systems for body-centric communication and the Internet of Things (IoT) requires the deployment of massive numbers of communication devices in everyday objects [1]. For esthetical reasons, such large numbers of electronic devices should be invisibly integrated. Moreover, for economical reasons, these wireless nodes should be extremely low cost. However, for practical and energy consumption reasons, they should exhibit high operating efficiency and large autonomy. The antenna system is typically a critical component when such goals must be met, as it is either large or highly inefficient. Moreover, its radiation performance may alter significantly in unfavorable deployment conditions.

Intensive research efforts have focused on novel dedicated antenna systems [2], [3], [4]. By reusing materials that are typically encountered in the object into which they are deployed, costs are reduced. By adopting a lowprofile antenna topology, even highly efficient antennas with dimensions comparable to the wavelength may be invisibly integrated. Moreover, by achieving wide band performance and by leveraging multi-antenna processing techniques, very high data rates may be achieved, thereby satisfying to specifications of various emerging wireless applications [1]. In this contribution, we outline the main challenges related to the design of such antenna systems, summarize design principles and provide techniques and guidelines to optimize performance while minimizing cost.

In Section II we discuss the design of antennas on offthe-shelf materials such as textile, paper, plastic, and cork. We outline the challenges related to replacing dedicated antenna substrates by materials typically encountered in the object into which the node is integrated. We also provide guidelines for the antenna designer to select optimal substrate materials. Next, In Section III we leverage Substrate Integrated Waveguide (SIW) technology to implement antennas in such materials. This enables designers to adopt antenna topologies with very high body-antenna isolation, which ensures very stable radiation characteristics in all kinds of adverse deployment conditions. Moreover, this isolation will avoid parasitic coupling between antenna and transceiver electronics in active antenna systems where the electronics are directly deployed onto the feed plane, as discussed in Section IV. Finally, in Section V we outline some multi-antenna processing techniques to achieve the throughput and reliability.

\section{NOVEL ANTENNA SUBSTRATE MATERIALS}

An interesting research path to cut costs consists in reusing materials already present available as substrates for the wireless nodes to be deployed in the IoT [4], [5] and personal wireless communication networks [5], [6]. Of course, substituting high-frequency laminates and dedicated antenna foams by common materials poses particular challenges to antenna design. First, they cannot rely on readily available data sheets. Instead, they need to rely on dedicated characterization procedures to characterize the dielectric characteristics and the losses of each potential substrate material [7], [8]. Second, they must account for large variations in material characteristics and substrate inhomogeneity [9]. Capturing these variability and uncertainty aspects calls for the inclusion of stochastic design frameworks in full-wave computer-aided design process. By providing insight into the probability density functions that characterize the variations in the antenna's figures of merit, these tools avoid unnecessary overspecification of the antenna's requirements, thereby reducing costs and potential electromagnetic compatibility issues. 
In recent years, antenna prototypes have been implemented on textiles, paper, plastic and cork [2], [5], [10]. Since most of these materials are flexible, it is paramount to verify to which extent bending affects the antenna performance [11]. Moreover, some of these substrates may absorb water particles, making the antenna performance dependent on the relative humidity conditions [12]. It is therefore paramount to select hydrophobice substrate materials, preferably exhibit moisture regains smaller than $3 \%$. Also compression may significantly alter the antenna performance [13]. To avoid permanent detuning after temporary compression of the substrate, it is advised to limit the compression set of the material to a value smaller than $30 \%$, verifying that satisfactory radiation performance is still obtained for a substrate height reduction of $70 \%$ of its nominal value [14].

\section{LOW-COST HIGH-PERFORMANCE SIW ANTENNAS}

Contrary to conventional wireless systems, antennas operating in a body-centric or an IoT setting will often be deployed in unfavorable operating conditions. Important deterioration in radiation efficiency may result from absorption by lossy materials located in objects in close proximity of the antenna, such as the human body or the "thing" serving as antenna platform. Therefore, adapted topologies with very high body-antenna isolation are needed to guarantee satisfactory performance in a varying operating conditions.

Recently, it has been shown that SIW technology may be implemented in the substrate materials described above to realize low-cost high-performance wide band antennas [2], [4], [10]. In an SIW cavity backed slot topology, the cavity shields the resonant fields from the antenna platform. Thereby, undesired radiation and parasitic surface waves are avoided. This significantly increases efficiency in unfavorable deployment conditions, since power is only radiated through the antenna slot, along the desired directions. Moreover, this topology allows for compact array design with minimal mutual coupling [15], [16]. SIW components may be realized by tubelets, thin wires, or perforations sealed by conductive epoxy [5].

Several SIW antenna designs for body-centric communication and the IoT have been proposed. By exciting multiple modes in the SIW antenna cavity, wide band operation is obtainable [10], [15], [17], [18]. By judiciously distributing the cavity modes of the intended frequency of operation, even ultra-wide band performance can be achieved [19]. Moreover, different types of passive microwave components may be implemented in the same type of technology, enabling a low-cost compact integration of the antenna array and its feed network [20].

\section{ACtive Antenna SYSTEMS}

Reliability is another aspect that must be included in the design process of body-centric antennas. Connectors and cables are often weak points, which easily break when exposed to stress, tension and torsion. This problem may be avoided by designing compact active antenna systems, where the large surface required by the highly efficient antenna system serves as an integration platform for the active electronic circuitry [21].

Owing to their excellent antenna/platform isolation, SIW antenna topologies enable direct deployment of both active and passive components on the antenna through a lowcost production process [5]. This enables the cost-effective realization of highly efficient, low-profile, autonomous wireless nodes. Active electronic circuits may also be deployed on other antenna topologies [22]. To avoid instability, it is important, however, to sufficiently shield the power and/or low-noise amplifier from the antenna radiation. This may be achieved by deployed the active circuits on the antenna feed plane, behind the ground plane. By connecting the amplifiers to the antenna with very short vias, losses and EMC issues are kept to a minimum. Moreover, the antenna and its active circuits may be cooptimized in a full-wave/circuit co-design process [23].

In a second step, the autonomy may be increased by directly positioning energy harvesters on the antenna plane [6], [18], [24], [25] and connected to the power management system. The DC wires must be kept away from the radiation edges of the antenna, in order not to modify the radiation pattern. In many antenna topologies, such as the SIW cavity backed slot antenna, the antenna plane is a DC ground plane, meaning that only one DC line must be routed to the feed plane. Hollow via holes may be exploited to bring the latter wire to the feed plane without affecting the antenna radiation.

\section{LEVERAGING MULTI-ANTENNA PROCESSING TECHNIQUES}

Unfavorable radio-wave propagation properties should also be considered. Often, shadowing and multi-path propagation will be more severe than in conventional wireless systems, due to the close proximity of the human body or other reflecting or absorbing objects. Moreover, the antenna orientation and position is highly uncertain. The use of multiple antennas, deployed at suitable locations, can significantly improve the reliability and capacity of the channel through MIMO techniques, such as beam forming [12], diversity [16], [26] and spatial multiplexing [27].

By combining signals originating from directional wearable antennas deployed at opposite locations of the human body, diversity can mitigate body shadowing and increase reliability. An even higher diversity gain may be achieved by also deploying multiple antennas at the access point and making use of space-time coding, provided a sufficiently reliable estimation of the MIMO channel is available at the receiver [26]. When very high data rates are required over short-distance wireless channels, antenna arrays may be 
deployed in smart furniture to provide ultra-small personal communication cells [15]. The combination of spatial multiplexing in very favorable channel conditions and the exploitation of the full system bandwidth for a single user provide an extremely large data throughput.

\section{CONCLUSION}

We have summarized some recent developments in the design of dedicated antenna systems for body-centric communication and the IoT. By reusing materials typically found in the application at hand, fabrication costs are kept low. Low-profile antenna topologies and SIW technology are used to obtain compact antennas than can be integrated invisibly and unobtrusively into their environment, with stable radiation characteristics in various operating conditions. Further improvement is achieved by co-optimizing the antenna with its active electronics, integrated onto the antenna feed plane to form an active antenna system. Multi-antenna processing techniques ensure reliable communication at all time and in all circumstances. Alternatively, they may be exploited for high-data rate communication in favorable channel conditions.

\section{ACKNOWLEDGMENT}

Sam Agneessens is a postdoctoral Fellow of the FWO-V.

\section{REFERENCES}

[1] H. Sundmaeker, P. Guillemin, P. Friess, and S. Woelffl, Eds., Vision and challenges for realising the internet of things. Luxembourg: Publications Office of the European Union, 2010.

[2] S. Agneessens and H. Rogier, "Compact half diamond dual-band textile hmsiw on-body antenna," IEEE Transactions on Antennas and Propagation, vol. 62, no. 5, pp. 2374-2381, May 2014.

[3] S. Agneessens, S. Lemey, T. Vervust, and H. Rogier, "Wearable, small, and robust: The circular quarter-mode textile antenna," IEEE Antenn. Wireless Propag. Lett., vol. 14, pp. 1482-1485, 2015.

[4] S. Lemey, O. Caytan, D. Vande Ginste, P. Demeester, H. Rogier, and M. Bozzi, "SIW Cavity-backed Slot (multi-)antenna Systems for the Next Generation IoT Applications," in IEEE Conference on Wireless Sensors and Sensor Networks, Austin, USA, Jan. 2016.

[5] M. Bozzi and R. Moro, "Low-cost fabrication, eco-friendly materials, and easy integration: the new technological paradigm for the future wireless sensor networks," in European Microwave Conference (EuMC), Nuremberg, Germany, Oct. 6-10, 2013.

[6] S. Lemey, F. Declercq, and H. Rogier, "Textile Antennas as Hybrid Energy-Harvesting Platforms," Proc. IEEE, vol. 102, no. 11, pp. 1833-1857, Nov. 2014.

[7] F. Declercq, H. Rogier, and C. Hertleer, "Permittivity and loss tangent characterization for garment antennas based on a new matrix-pencil two-line method," IEEE Trans. Antennas Propagat., vol. 56, no. 8, pp. 2548-2554, Aug 2008.

[8] F. Declercq, I. Couckuyt, H. Rogier, and T. Dhaene, "Environmental High Frequency Characterization of Fabrics Based on a Novel Surrogate Modelling Antenna Technique," IEEE Trans. Antennas Propagat., vol. 61, no. 10, pp. 5200-5213, Oct. 2013.

[9] M. Rossi, A. Dierck, H. Rogier, and D. Vande Ginste, "A Stochastic Framework for the Variability Analysis of Textile Antennas," IEEE Trans. Antennas Propagat., vol. 62, no. 12, Dec 2014.

[10] O. Caytan, S. Lemey, S. Agneessens, D. Vande Ginste, P. Demeester, C. Loss, R. Salvado, and H. Rogier, "Half-mode substrateintegrated-waveguide cavity-backed slot antenna on cork substrate," Antennas Wirel. Propag. Lett., vol. PP, no. 99, 2015.
[11] F. Boeykens, H. Rogier, and L. Vallozzi, "An efficient technique based on polynomial chaos to model the uncertainty in the resonance frequency of textile antennas due to bending," IEEE Trans. Antennas Propagat., vol. 62, no. 3, pp. 1253-1260, 2014.

[12] M. Scarpello, D. Vande Ginste, and H. Rogier, "Design of a Lowcost Steerable Textile Antenna Array Operating in Varying Relative Humidity Conditions.” Microw. Opt. Technol. Lett., vol. 54, no. 1, pp. 40-44, Jan. 2012.

[13] M. Rossi, S. Agneessens, H. Rogier, and D. V. Ginste, "Stochastic Analysis of the Impact of Substrate Compression on the Performance of Textile Antennas," IEEE Trans. Antennas Propagat., vol. 64, no. 6, pp. 2507-2512, Jun 2016.

[14] C. Hertleer, H. Rogier, L. Vallozzi, and L. Van Langenhove, "A Textile Antenna for Off-Body Communication Integrated Into Protective Clothing for Firefighters," IEEE Trans. Antennas Propagat., vol. 57, no. 4, pp. 919-925, Apr 2009.

[15] S. Lemey, T. Castel, P. V. Torre, T. Vervust, J. Vanfleteren, P. Demeester, D. Vande Ginste, and H. Rogier, "Threefold Rotationally Symmetric SIW Antenna Array for Ultra-Short-Range MIMO Communication," IEEE Trans. Antennas Propagat., vol. 64, no. 5, pp. 1689-1699, May 2016.

[16] T. Castel, S. Lemey, P. Van Torre, C. Oestges, and H. Rogier, "FourElement Ultrawideband Textile Cross Array for Dual-Spatial and Dual-Polarization Diversity," IEEE Antenn. Wireless Propag. Lett., vol. 16, pp. 481-484, 2017.

[17] G. Q. Luo, Z. F. Hu, W. J. Li, X. H. Zhang, L. L. Sun, and J. F. Zheng, "Bandwidth-Enhanced Low-Profile Cavity-Backed Slot Antenna by Using Hybrid SIW Cavity Modes," IEEE Trans. Antennas Propagat., vol. 60, no. 4, pp. 1698-1704, Apr. 2012.

[18] S. Lemey, F. Declercq, and H. Rogier, "Dual-Band Substrate Integrated Waveguide Textile Antenna With Integrated Solar Harvester," IEEE Antenn. Wireless Propag. Lett., vol. 13, no. 1, 2014.

[19] S. Lemey and H. Rogier, "SIW textile antennas as a novel technology for UWB RFID tags," in 2014 IEEE RFID-TA, Tampere, Finland, Sept. 8-9, 2014, pp. 256-260.

[20] R. Moro, S. Agneessens, H. Rogier, A. Dierck, and M. Bozzi, "Textile Microwave Components in Substrate Integrated Waveguide Technology," IEEE Trans. Microw. Theory Techn., vol. 63, no. 2, pp. 422-432, Feb 2015.

[21] A. Dierck, S. Agneessens, F. Declercq, B. Spinnewyn, G.-J. Stockman, P. Van Torre, L. Vallozzi, D. Vande Ginste, J. Vanfleteren, T. Vervust, and H. Rogier, "Active Textile Antennas in Professional Garments for Sensing, Localisation and Communication," International Journal of Microwave and Wireless Technologies, vol. 6, no. 3-4, pp. 331-341, 2014.

[22] A. Dierck, H. Rogier, and F. Declercq, "An Active Wearable Dualband Antenna for GPS and Iridium Satellite Phone Deployed in a Rescue Worker Garment," in IEEE Int. Conf. on RFID Technologies and Applications, 4-5 Sept. 2013, pp. 1-5.

[23] A. Dierck, F. Declercq, and H. Rogier, "Review of active textile antenna co-design and optimization strategies," 2011 IEEE International Conference on RFID-Technologies and Applications, pp. 194-201, Sep 15-16 Sept., 2011.

[24] S. Lemey, S. Agneessens, P. Van Torre, K. Baes, J. Vanfleteren, and H. Rogier, "Wearable Flexible Lightweight Modular RFID Tag With Integrated Energy Harvester," IEEE Trans. Microw. Theory Techn., vol. 64, no. 7, pp. 2304-2314, Jul 2016.

[25] O. Caytan, S. Lemey, S. Agneessens, and H. Rogier, "SIW antennas as hybrid energy harvesting and power management platforms for the internet of things," International Journal of Microwave and Wireless Technologies, vol. 8, no. 4-5, pp. 767-775, Mar 2016.

[26] P. Van Torre, L. Vallozzi, C. Hertleer, H. Rogier, M. Moeneclaey, and J. Verhaevert, "Indoor Off-Body Wireless MIMO Communication With Dual Polarized Textile Antennas," IEEE Trans. Antennas Propag., vol. 59, no. 2, pp. 631-642, Feb 2011.

[27] T. Castel, P. Van Torre, L. Vallozzi, M. Marinova, S. Lemey, W. Joseph, C. Oestges, and H. Rogier, "Capacity of Broadband Body-to-Body Channels between Firefighters wearing Textile SIW Antennas," IEEE Trans. Antennas Propagat., vol. 64, no. 5, pp. 1918 - 1931, 2016. 